\title{
Infección intrahospitalaria a pseudomonas aeruginosa en un servicio de Pediatría. Tipificación por producción de Piocina *
}

HERIBERTO FERNANDEZ J. ** y CYNTIA LEMBKE P. **

La constitución de Pseudomonas aeruginosa en uno de los agentes etiológicos más frecuentes de infecciones intrahospitalarias ha sido consecuencia de la natural resistencia que presenta este microorganismo a la mayoría de los antibióticos y quimioterápicos y al desplazamiento de algunas bacterias, - también responsables de estas infecciones - por la constante presión selectiva ejercida por las drogas antimicrobianas dentro del hospital (1), (2), (3), (4).

En un centro asistencial, los servicios destinados a la atención de pacientes quemados, prematuiros, recién nacidos y pediátricos, son las áreas de mayor riesgo y propensión a sufrir el impacto de estas infecciones (5), (6), (7), (8), (9), (10), (11), (12).

Actualmente, gracias a los métodos de clasificación desarrollados para Ps. aeruginosa (13), (14), (15), (16), es posible establecer el trazado epidemiológico de muchos brotes infecciosos, que, una vez conocidas sus fuentes y vías de diseminación, son susceptibles de controlar.

Empleando la capacidad productora de piocina de $P$ s. aeruginosa para su tipificación, hemos tratado de establecer la epidemiología de un brote infeccioso intrahospitalario producido por este microorganismo en el Servicio de Pediatría del Hospital J. Kennedy de Valdivia.

MATERIAL Y METODO.- Se identifican, según los esquemas del Manual de Bergey (7), y de Gilardi, (18), 51 cepas de Ps. aeruginosa aisladas durante un brote infeccioso intrahospitalario ocurrido en el Servicio de Pediatría del Hospital J. Kennedy de Valdivia en el segundo trimestre de 1971.

La procedencia y el número de cepas aisla-

\footnotetext{
* Financiamiento otorgado por la Vice-Rectoría de Investigaclón. Universidad Austral de Chile - Valdivia - (Chile). Trabajo de Ingreso a la Saciedad Chilena de Pediatría, presentado en las IV Jornadas Anuales de Pediatrfa, Valdivia, Octubre, 1974.

* División de Microblología Clf́nica, Facultad de Medicina. Universidad Austral de Chile, Valdivia.
}

das, por muestra, quedan establecidos en la Tabla I. De ellas, 37 fueron aisladas de muestras de origen clínico obtenidas de sendos pacientes que presentaban cuadros infecciosos activos. Las 14 cepas restantes fueron aisladas luego de efectuar un control bacteriológico de la cocina de leche que incluía la búsqueda de $P$ s. aeruginosa en muestras de deposiciones, manos y uñas de los dos manipuladores de alimentos que trabajaban en ella.

Las muestras clínicas fueron sembradas en agar sangre, agar SS o Mc. Conkey y en tubo de caldo thioglicolato, utilizándose agar cetrimida y agar sangre para la búsqueda dirigida de $P s$. aeruginosa en el ambiente, mamaderas, leche y en muestras obtenidas del personal del Servicio Dietético de Leche (Cocina de Leche).

La tipificación de las cepas aisladas se hizo de acuerdo al método desarrollado por Gillies y Govan (15), (16), con algunas modificaciones (19).

Resultados.- De las 51 cepas de Ps. aeruginosa sometidas a la piocinotipia, 50 correspondieron al tipo 1 y sólo una de ellas, por su incapacidad de inhibir el desarrollo de alguna de las cepas indicadoras, no produjo un patrón de inhibición que permitiera su encasillamiento en uno de los 37 tipos propuestos por Gillies y Govan. Esta cepa, que hemos denominado no clasificable, fue aislada de pus de herida.

De las 50 cepas tipo 1 sometidas a la subtipificación, 48 produjeron un patrón de inhibición que permitió su clasificación dentro del subtipo d. Los patrones de inhibición de las 2 cepas restantes no concordaban con ninguno de los 8 subtipos propuestos por estos autores, siendo consideradas como Ps. aeruginosa tipo 1 subtipo no clasificable. Ambas cepas fueron aisladas de deposiciones, una proveniente del Servicio de Pediatría y la otra, del Servicio de Prematuros. 
Discusión - De los resultados obtenidos con la piocinotipia se desprende que el agente etiológico responsable de este brote infeccioso fue la misma cepa de PS. aeruginosa, que, de acuerdo a su piocinogenia corresponde al tipo 1 subtipo d.

Por otra parte, la revisión de las fichas clínicas permitió constatar que todos los pacientes adquirieron la infección dentro de un período que sobrepasaba las primeras $48 \mathrm{hrs}$. de hospitalización, hecho considerado por Pien y col. como índice de infección intrahospitalaria (20).

El aislamiento de la misma cepa de Ps. aeruginosa a partir de mamaderas, leche, mesones y personal del Servicio Dietético de Leche (Tabla I), están confirmando la presunción de clínicos y enfermeras que señalaban a este Servicio como fuente de origen de esta epidemia. El hallazgo de Ps. aeruginosa tipo 1 subtipo d en dos pacientes del Servicio de Prematuros, en la misma época, agrega otra prueba en favor de esta presunción, ya que, ambos servicios -Pediatría y Prematuroscompartían la cocina de leche.

La utilización de la capacidad productora de piocina de $P s$. aeruginosa como marcador epidemiológico nos ha permitido establecer sus posibles reservorios y vías de diseminación en este brote infeccioso intrahospitalario (Fig. 1).

Uno de los manipuladores de alimentos del Servicio Dietético de Leche - portador sano de Ps. aeruginosa (Tabla I) - habría sido el reservorio y primer eslabón de la cadena. La condición de portador intestinal de este microorganismo es un fenómeno relativamente frecuente. Algunos autores señalan que de un 11 a un $15 \%$ de la población normal lleva el germen en su intestino (21). La utilización de Ps. aeruginosa como índice de contaminación fecal está demostrando, por otra parte, su alta incidencia en las deposiciones humanas (22).

\section{Figura 1}

Posible reservorio y vías de diseminación de Ps. aeruginosas en el brote infeccioso estudiado.

Reservorio

Tracto intestinal manipulador 1

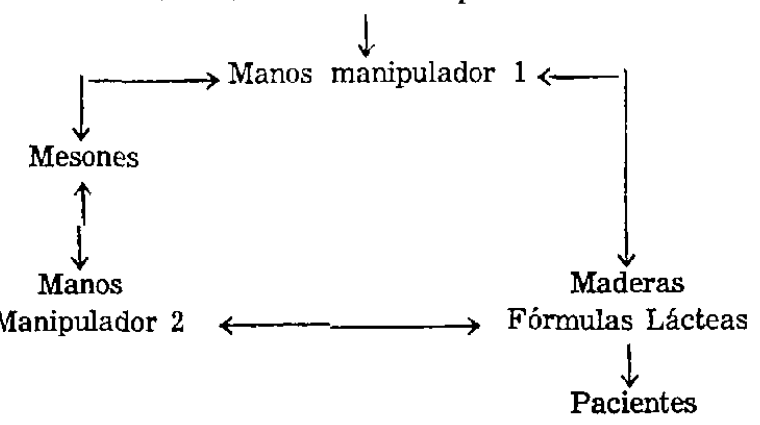

\section{TABLA I}

Procedencia, tipo de muestra y número de cepas de $P$ s. aeruginosa aisladas en un brote infeccioso intrahospitalario ocurrido en un Servicio de Pediatría.

\begin{tabular}{llc}
\hline \multicolumn{1}{c}{ Muestra } & Servicio procedencia & N $^{\circ}$ Cepas \\
\hline Deposiciones & Pediatría & 19 \\
Deposiciones & Prematuros & 3 \\
Deposiciones & Cocina de leche & 1 \\
& $\quad$ (Manipulador) & \\
Orina & Pediatría & 4 \\
Pus herida & Pediatría & 3 \\
L. C. R. & Pediatría & 3 \\
Pus oído & Pediatría & 2 \\
Secr. traqueal & Pediatría & 2 \\
Pus pleural & Pediatría & 1 \\
Mamaderas & Cocina de leche & 6 \\
Mesones & Cocina de leche & 2 \\
Leche & Cocina de leche & 3 \\
Manos y uñas & Cocina de leche & 2 \\
\multicolumn{2}{c}{$\quad$ (manipuladora) } \\
\hline TOTAL DE CEPAS AISLADAS & 51 \\
\hline
\end{tabular}

Las manos de este portador, contaminadas seguramente por malos hábitos higiénicos, habrían llevado la $P s$, aeruginosa hasta los mesones, mamaderas y fórmulas lácteas. Estos, a su vez, actuarían como fuente de infección para las manos del segundo portador, que, constituídas en vector mecánico del germen, habrían contaminado también, mesones, mamaderas y fórmulas lácteas.

$\mathrm{La}$ ingestión de alimentos contaminados con $P$ s. aeruginosa -en este caso la leche- permite que esta bacteria pueda llegar a formar parte de la flora intestinal. En individuos susceptibles, la colonización intestinal puede desencadenar en una infección, especialmente en aquellos pacientes que sufren procesos anergizantes. La existencia de un paciente con un cuadro infeccioso activo trae consigo otro problema: la transmisión del microorganismo a otros pacientes a través de las manos del personal asistencial (23).

Los hallazgos bacteriológicos efectuados y las conclusiones epidemiológicas a que hemos llegado, ponen de manifiesto, una vez más, la importancia del Servicio Dietético de Leche, en la salud del niño. Por otra parte, la participación de la leche, mamadera y bombas lácteas como reservorios y vectores de Ps. aeruginosa y otras bacterias (24), (25), exige periódicos y continuos controles microbiológicos en este Servicio, que, junto a su adecuado funcionamiento y al empleo de estrictas técnicas de antisepsia, contribuirán, en gran medida, a la obtención de fórmulas lácteas sanitariamente aptas. 


\section{RESUMEN}

Se tipifican, de acuerdo a su capacidad productora de piocina, 51 cepas de Pseudomonas aeruginosa aisladas durante un brote infeccioso intrahospitalario ocurrido en el Servicio de Pediatría del Hospital J. Kennedy de Valdivia (Chile) en el segundo trimestre de 1971.

Cincuenta cepas correspondieron al tipo 1 de Gillies y Govan y 48 de éstas al subtipo d.

Empleando la piocinotipia como marcador epidemiológico fue posible demostrar que el agente etiológico de esta infección intrahospitalaria fue la misma cepa de Ps. aeruginosa (tipo 1 d), como también, señalar sus posibles reservorios y vías de diseminación (Fig. 1).

\section{AbSTRACT}

Pyocine-typing was done on 51 strains of Pseudomonas aeruginosa isolated during a nosocomial infectious outbreak ocurred in the Paediatric Service of the John Kennedy's Hospital (Valdivia, Chile).

50 strains were typified as type 1 from Gillies and Govan and 48 of them, as subtype d.

Employing the pyocine - typing method from Gillies and Govand as an epidemiological marker, it was possible to determine that the same strain (type 1, subtype d) of Ps. aeruginosa was the aethyological agent of this nosocomial outbreak, and it was also possible to point out his reservoir and spreading vias (Fig. 1).

\section{REFERENCIAS}

1.- Farmer III, J. J. \& Herman, L. G.- Epidemiological fingerprinting of Pseudomonas aeruginosa by the Production of and Sensitivity to Pyocin and Bacteriophage. Applie Microbiology 18: $760-765$, 1969.

2.-Heckman, M. G.; Babcock, M. G. \& Rose, H. D.Pyocine Typing of Pseudomonas aeruginosa: Clinical and Epidemiological Aspects. American Journal of Clinical Pathology. 57: $35-42,1972$.

3.- Adler, J. L. \& Finland, M.- Susceptibility of Recent Isolated of Pseudomonas aeruginosa to Gentamicin, Polymixin and Five Penicillins, with Observations on the Pyocin and Immunotypes of the Strains. Applied Microbiology 22: 870-875, 1971.

4.-Dámaso, D.- Estudio epidemiológico de Pseudomonas aeruginosa. Piocinotipia en la Clínica "Puerta de Hierro". Revista de Sanidad e Higiene Pública 45: $1065-1078,1971$.
5.-Edmonds, P.; Suskind, R. R.; MacMillan, B. G. \& Holder, I. A.- Epidemiology of Pseudomonas aeruginosa in a Burns Hospital: Evaluation of Serological, Bacteriophage and Pyocin Typing Methods. Applied Microbiology 24: 213 - 218, 1972.

6.-Edmonds, P.; Suskind, R. R.; MacMillan, B. G. \& Holder, I. A.- Epidemiology of Pseudomonas aeruginosa in a Burns Hospital: Surveillance by a Combined Typing System. Applied Microbiology 24: $219-225,1972$.

7.-Kominos, S. D.; Copeland, C. E. \& Grosiak, B.Mode of Transmission of Pseudomonas aeruginosa in a Burn Unit and an Intensive Care Unit in a General Hospital. Applied Microbiology 23: 309312, 1972.

8.-Vega-Franco, L.; Vásquez. V. \& Ruiz-Izaguirre, R.- Prevalencia de portadores de Pseudomonas aeruginosa en niños hospitalizados. Revista Latinoamericana de Microbiología 12: 159-168, 1970.

9.-Abbe, J. S. \& Moffet, H. L.- Surveillance of Pseudomonas aeruginosa infections in a Children's Hospital. Antimicrobial Agents and Chemotherapy. pp. $303-308,1970$.

10.-Tagg. J. R. \& Mushin, R.-- Epidemiology of Pseudomonas aeruginosa infection in hospitals. 1.Pyocine typing of Ps. aeruginosa. The Medical Journal of Autralia. 1: $847-852,1971$.

11.-Deighton, M. A.; Tagg. J. R. \& Mushin, R.- Epidemiology of Pseudomonas aeruginosa infection in hospitals. 2. Fingerprinting of Ps. aeruginosa strains in a study of cross - infection in a Children's Hospital. The Medical Journal of Australia 1: 892 . 896, 1971.

12.-Bobo, R. A.; Newton, E. J.; Jones, L. F.; Farmer, L. H. \& Farmer III, J. J.-- Nursery Outbreak of Pseudomonas aeruginosa: Epidemiological Conclusions from Five Different Typing Methods. Applied Microbiology 25: 414-420, 1973.

13.-Sjöberg, L. \& Lindberg. A. A.- Phage typing of Pseudomonas aeruginosa. Acta Pathologica et Microbiologica Scandinavica 74: 61-68, 1968.

14.-Moody, M. R.; Young, V. M.; Kenton, D. M. \& Vermeulen, G. D. Pseudomonas aeruginosa in a Center for Cancer Research. I. Distribution of Intraspecies Types from Human and Environmental Sources. The Jornal of Infectious Diseases 125: 95 - 101, 1972.

15.-Gillies, R. \& Govan, J.- Typing of Pseudomonas pyocynea by pyocine production. Journal of $\mathrm{Pa}$ thology and Bacteriology 91: 339-345, 1966. 
16.-Govan, J. \& Gillies, R.- Further studies in the pyocine typing of Pseudomonas pyocyanea. Journal of Medical Microbiology 2: 17-25, 1969.

17.-Breed, R. S.; Murray, E. G. D. \& Smith, N. R. Manual of Determinative Bacteriology, $7^{\mathrm{a}} \mathrm{Ed} ., \mathrm{Bal}$ timore, The Williams and Wilkins Co., 1957.

18.- Gilardi, G. L.- Characterization of Pseudomonas Species Isolated from Clinical Specimens. Applied Microbiology 21: $414-419,1971$.

19.- Fernández, H. \& Lembke, C.- Blatella germanica (cucaracha) Vector intrahospitalario de Pseudomonas aeruginosa. Boletín del Instituto Bacteriológico de Chile 15: 21 - 23, 1973.

20.-Pien, F. D.; Martin, W. J.; Hermans, P. E. \& Washington II, J. A.- Clinical and Bacteriologic Observations on the Proposed Species, Enterobacter agglomrans (The Herbicola - Lathyri Bacteria). Mayo Clinic Proceedings 45: 739-745, 1972.
21.-Ruscke, R.-Pseudomonas aeruginosa - "ein Zivilisationskeim"--. Nachweis seiner Vermeehrung in Kationenaustauschern von Gescirrspuelmaschinen. Zentral blatt für Bakteriologie und Hygien, I. Abteilung, Originale B 156: $391-398,1972$

22.-Némedi, L. \& Lányi, B.- Incidence and Hygienic Importance of Pseudomonas aeruginosa in Water. Acta Microbiologica. Academiae Scientiarum Hungaricae 18: $319-326,1971$.

23.-Kominos, S. D.; Copeland, C. E.; Grosiak, B. \& Postic, B.- Introduction of Pseudomonas aeruginosa into a Hospital Via Vegetables. Applied Microbiology 24: $567-570,1972$.

24.-Ayliffe, G. A. J.; Collins, B. J. \& Petit, F.Contamination of Infant Feeds in a Milton Milk Kitchen. The Lancet 1: 559 - 560, 1970

25.-Thom, A. R.; Cole, A. P. \& Watrasiewicz, K.Pseudomonas aeruginosa infection in a neonatal nursery, possibly transmitted by a Breast-milk pump. The Lancet 1: 560-561, 1970. 